\title{
Relationship between ferroelectricity and Dzyaloshinskii-Moriya interaction in multiferroics and the effect of bond-bending
}

\author{
C. D. $\mathrm{Hu}$ \\ Department of Physics and Center for Theoretical Sciences, National Taiwan University, Taipei, Taiwan 10617, Republic of China
}

(Received 1 November 2007; revised manuscript received 13 April 2008; published 12 May 2008)

\begin{abstract}
We studied the microscopic mechanism of multiferroics, in particular, with the "spin current" model [H. Katsura et al., Phys. Rev. Lett. 95, 057205 (2005)]. Starting from a system with helical spin configuration, we solved for the forms of the electron wave functions and analyzed their characteristics. The relation between ferroelectricity and Dzyaloshinskii-Moriya interaction [I. Dzyaloshinskii, J. Phys. Chem. Solids 4, 241 (1958) and T. Moriya, Phys. Rev. 120, 91 (1960)] is clearly established. There is also a simple relation between the electric polarization and the wave vector of magnetic orders. Finally, we show that the bond-bending existing in transition metal oxides can enhance ferroelectricity.
\end{abstract}

DOI: 10.1103/PhysRevB.77.174418

PACS number(s): 75.80.+q, 77.80.-e

\section{INTRODUCTION}

Experimental findings ${ }^{1-4}$ in recent years have revived the interest in multiferroics. They showed that magnetic and ferroelectric orders are closely related. ${ }^{5-8}$ What is more intriguing is that only certain types of magnetic orders, namely, helical spins and frustrated spins, can be coupled to ferroelectricity. ${ }^{9}$ It is this fascinating interplay between ferroelectric and magnetic orders that has attracted many researchers. There were already models based on the Ginzburg-Landau theory ${ }^{10,11}$ that provide instructive physical description of the systems. As for the microscopic mechanism, there are currently two schools of theories. One of them proposed that the electric polarization and the anomaly of dielectric constant come from atomic displacements. The displacements or phonons are, in turn, coupled to spins. ${ }^{12-14}$ Although proposed for systems of orthorhombic structure, it is more readily applied to multiferroics of hexagonal structures, such as $\mathrm{HoMnO}_{3}$, as there is experimental evidence of atomic displacements from neutron scattering data. ${ }^{15}$ The second school of theory proposed a new possibility: electric polarization coming from electronic wave function and thus density distribution. Katsura, Nagaosa, and Balatsky $(\mathrm{KNB})^{16}$ predicted that the magnetoelectric effect can be induced by "spin current." 17 The coupling between spin current and internal electric field has the same form as that of Dzyaloshinskii-Moriya (DM) interaction ${ }^{18,19}$ or ac effect ${ }^{20}$ where the motion of a magnetic moment is coupled to electric field. In this latter theory, the atomic displacement is not essential. On the other hand, spin-orbit interaction is indispensable in generating electric dipole moments.

The spin current model, although a bright idea, needs additional substantiation in order to be applied to physical systems. Jia et al. $^{21}$ gave a detailed calculation of this model. Their results showed that the spin current model is able to explain at least semiquantitatively many experimental data. This model was also applied to the systems with $e_{g}$ orbitals such as $\mathrm{TbMnO}_{3}{ }^{22}$ In our opinion, the foremost task is the embodiment of this idea in a crystal in which completely different properties and behaviors can emerge from complexities and interrelations between electrons, spins, and lattice structure. Equally important is the calculation of the magnitude of electric polarization induced by spin current. According to $\mathrm{KNB}$, the polarization is of the orders $e I(t / \Delta)$ or $e I(t / \Delta)^{3}$ for one or two holes. Here, $I$ is the expectation value of length, $t$ is the hybridization energy, and $\Delta$ is the energy difference between $d$ orbitals and $p$ orbitals. According to their estimation, $t=V(p d \pi) \sim 0.1 \mathrm{eV}, \Delta \approx 2 \mathrm{eV}$, and $e \mathrm{I} / a^{3} \sim 10^{4} \mu \mathrm{C} / \mathrm{m}^{2}$, where $a$ is the lattice constant. One can see that the magnitude is compatible with experimental data only in very favorable conditions. Therefore, it is desirable to conceive possible and realistic mechanism to enhance the ferroelectricity-magnetism coupling.

KNB derived an elegant expression for the electric polarization of a three-atom system $\vec{P} \sim e I \hat{e}_{12} \times\left(\hat{e}_{1} \times \hat{e}_{2}\right)$, where $\hat{e}_{1}$ and $\hat{e}_{2}$ are the directions of spins of the transition metal ions and $\hat{e}_{12}$ is the bond direction. We shall see how the expression conforms in a crystal and how it is related to the wave vector of the helical magnetic order. We will explain the reason why it is advantageous to have the helical spin configuration for ferroelectricity and also elaborate the roles played by the spin-orbit interaction. In fact, we will show that the electric polarization directly comes from DM interaction. Finally, we point out that a common feature in transition metal oxides, which is the bond-bending, can enhance electric polarization.

\section{DESCRIPTION OF THE SYSTEM}

We are going to consider two features in the system: the helical spin configuration and bond-bending. Mostovoy ${ }^{23}$ studied a system of degenerate double-exchange interaction and next-nearest-neighbor hopping. He found that under certain conditions, the helical spin configuration is stable. Thus, we shall take that as our starting point. The transition metal ion, with position vector $\vec{R}_{j, m}=\vec{R}_{j}+\vec{r}_{m}$, where $\vec{R}_{j}$ is the position vector of the $j$ th lattice point and $\vec{r}_{m}$ is the position vector of the $m$ th ion in the basis, has the following form for its spin (presumably those of $t_{2 g}$ electrons):

$$
\vec{S}_{j, m}=S\left[\left(\hat{e}_{x} \cos \phi+\hat{e}_{y} \sin \phi\right) \sin \left(\vec{q} \cdot \vec{R}_{j, m}\right)+\hat{e}_{z} \cos \left(\vec{q} \cdot \vec{R}_{j, m}\right)\right] .
$$

The helical spin order has a wave vector $\vec{q}$. As shown in Fig. 1 , the projection of spins on $x y$ plane makes a fixed angle $\phi$ with the $x$ axis. 


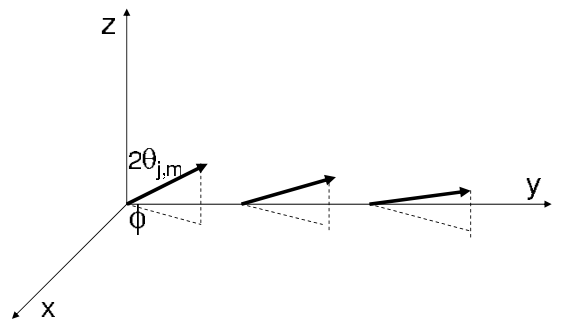

FIG. 1. Schematic helical spin configuration. $\phi$ is the angle between the projections of spins on the $x y$ plane and $x$ axis. The angle between spins and the $z$ axis is twice of $\theta_{j, m}=\vec{q} \cdot \vec{R}_{j, m} / 2$ defined in text.

Hund's coupling in the transition metal ions, $-J_{H} \Sigma \vec{S}_{j, m} \cdot \vec{s}_{j, m}$, is the dominant mechanism in the system. If the local spins in Eq. (1) are treated as classical spins, their effect on the spins of hybridizing electrons, denoted by $\vec{s}_{j, m}$, is equivalent to an effective magnetic field. Thus, the eigenstate of the spin of a hybridizing electron on the $m$ th transition metal ion with lower energy is

$$
\left(\begin{array}{c}
\cos \theta_{j, m} \\
e^{i \phi} \sin \theta_{j, m}
\end{array}\right)
$$

where $\theta_{j, m}=\vec{q} \cdot \vec{R}_{j, m} / 2$. This will affect the hybridization of orbitals.

Next, we consider the hybridization. KNB studied a transition metal (TM)-O-TM three-atom triad. Due to the symmetries of orbitals, the hybridization arises from $\pi$ bonding. If the TM-O-TM triad is not linear [illustrated in Fig. 2(a) where $\alpha$ is the bond angle], the $p_{x}$ orbitals of oxygen (if the bond is approximately in the $x$ direction) can also take part in electron transfer, where $\sigma$ bonding can be realized and the
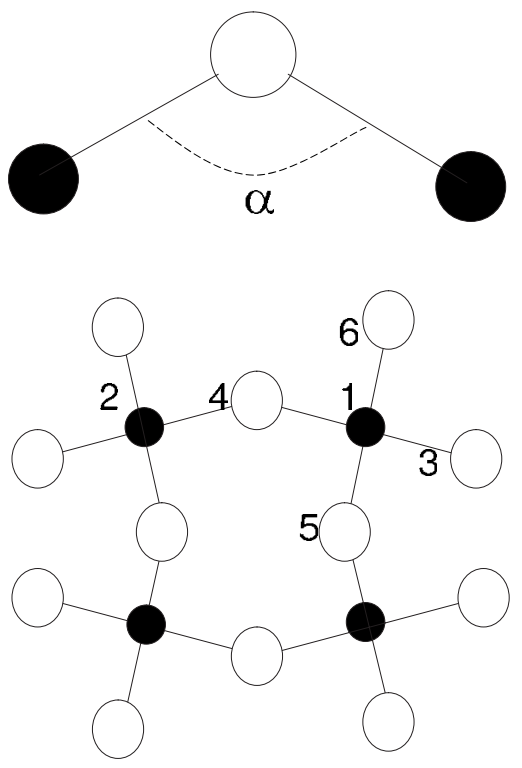

FIG. 2. (a) Bond angle $\alpha$. (b) A two-dimensional lattice with bond-bending. The solid dots and circles denote the transition metal ions and oxygen atoms, respectively. The atoms are labeled so as to facilitate later deduction. hybridization energy can be greater. In fact, the bondbending occurs quite often in transition metal oxides (see, for example, Ref. 24). The $a b$ plane of the crystal is shown in Fig. 2(b) where the solid dots and circles denote the transition metal ions and oxygen atoms, respectively. There are two distinct transition metal ions in the basis, and thus $m$ $=1,2$, due to bond-bending. As a result, two transition metal ions and four oxygen atoms form the basis of the crystal, which is enlarged by bond-bending. The atoms are labeled so as to facilitate later deduction. As mentioned above, bondbending will affect the hybridization energies. They can be found in Slater and Koster. ${ }^{25}$

Bond-bending has other profound effects. It changes the symmetry of the surrounding of atoms and thus the symmetry of crystal field. In an orthorhombic crystal $(a \neq b)$ with bond-bending, the $x y$ orbitals of the transition metal ion will mix with the $x^{2}-y^{2}$ orbitals. The resulting orbital has the form

$$
\cos \beta|x y\rangle \mp \sin \beta\left|x^{2}-y^{2}\right\rangle,
$$

where the \pm sign is determined by the direction of the displacement of the oxygen atom away from the line joining two transition metal ions. $\beta$ and $\alpha$ are of the same order of magnitudes. Ideally, if the crystal field is determined by the four nearest oxygen ions on the $a b$ plane, then $\beta=\alpha^{\prime}+\delta \alpha^{\prime}$, where $\alpha^{\prime}=\pi-\alpha$. The deviation $\delta \alpha^{\prime}$ is due to the fact that the symmetry of crystal field is not determined by bond-bending alone. We expect $\delta \alpha^{\prime}$ to be finite but smaller than $\alpha$ in orthorhombic crystals of manganites and large in compounds such as $\mathrm{Ni}_{3} \mathrm{~V}_{2} \mathrm{O}_{8}$. However, to get its magnitude, one needs to carry out a very precise first-principles calculation, which is beyond the scope of the current paper.

The hybridization of the $p$ orbital of oxygen atoms and the $x y$ orbitals of transition ions is equal to $\pm(\sqrt{3} / 2) \sin \alpha \sin (\alpha / 2) V(p d \sigma)$, where the sign is again determined by the direction of the displacements of oxygen atoms. That of the $p$ orbital of oxygen atoms and the $x^{2}-y^{2}$ orbitals of transition ions is equal to $-(\sqrt{3} / 2) \cos \alpha \sin (\alpha / 2) V(p d \sigma)$. Although the $x^{2}-y^{2}$ orbitals have greater hybridization energy, its amplitude is smaller as one can see from Eq. (3). The resulting hybridization energy is $\pm(\sqrt{3} / 2) \sin \left(\alpha^{\prime}-\beta\right) \sin (\alpha / 2) V(p d \sigma)$. We note in passing that since $\alpha$ is close to $\pi$, if $\left|\beta-\alpha^{\prime}\right| \approx \pi / 12$, then $(\sqrt{3} / 2) \sin \left(\alpha^{\prime}-\beta\right) \sin (\alpha / 2) V(p d \sigma) \approx 0.2 V(p d \sigma)$, which is often greater than $V(p d \pi)$.

\section{CALCULATION}

Due to the Hund coupling, the spins of the hybridizing electron are projected to the local spins and the hybridization energy is modified. It is the main part of our Hamiltonian. We considered $\sigma$ bond only. Hence, the orbitals involved are that in Eq. (3) of transition metal ions, $p_{x}$ orbitals of oxygen atoms 3 and 4 , and $p_{y}$ orbitals of oxygen atoms 5 and 6 , where the numerals are shown in Fig. 2(b). Later, we take the spin-orbit interaction, and more orbitals, into account. It will be treated as a perturbation. Our Hamiltonian thus has two parts, $H=H_{0}+H_{1}$ : 


$$
\begin{aligned}
H_{0}= & \sum \varepsilon_{p} c_{p i l, \sigma}^{\dagger} c_{p i l, \sigma}+\sum \varepsilon_{d} c_{d j m}^{\dagger} c_{d j m}-\sum_{n n} V(-1)^{l} \\
& \times\left[\cos \theta_{j, m} c_{d j m}^{\dagger} c_{p i l, \uparrow}+e^{i \phi} \sin \theta_{j, m} c_{d j m}^{\dagger} c_{p i l, l}\right]+\text { H.c. }
\end{aligned}
$$

and

$$
H_{1}=\lambda \sum_{j, m} \vec{l}_{j, m} \cdot \vec{s}_{j, m}
$$

where the hybridization energy $V=(\sqrt{3} / 2) \sin (\beta$ $\left.-\alpha^{\prime}\right) \sin (\alpha / 2) V(p d \sigma)$ is only among nearest neighbors. $\lambda$ is the strength of spin-orbit coupling. $\varepsilon_{p}, \varepsilon_{d}$ and $c_{p i l, \uparrow(\downarrow)}$, and $c_{d j m}$ are the energies and field operators of the $p$ orbitals in oxygen atoms and $d$ orbitals in transition metal ions. $l$ and $m$ are the indices of oxygen atoms and transition metal ions in the basis. As shown in Fig. 3, $m=1,2$ for manganese ions and $l=3-6$ for oxygen atoms. As previously mentioned, the localized spins were approximated as an effective magnetic field of angles $\theta_{j, m}$ and $\phi$. We now made the following transformation:

$$
\begin{aligned}
& p_{i l, \uparrow}=\cos \left(\vec{q} \cdot \vec{R}_{i, l} / 2\right) c_{p i l, \uparrow}+e^{i \phi} \sin \left(\vec{q} \cdot \vec{R}_{i, l} / 2\right) c_{p i l, \downarrow}, \\
& p_{i l, \downarrow}=\cos \left(\vec{q} \cdot \vec{R}_{i, l} / 2\right) c_{p i l, \downarrow}-e^{-i \phi} \sin \left(\vec{q} \cdot \vec{R}_{i, l} / 2\right) c_{p i l, \uparrow},
\end{aligned}
$$

where $\vec{R}_{i, l}=\vec{R}_{i}+\vec{r}_{l}$, with $\vec{r}_{l}$ being the position vector of the $l$ th oxygen atom in the basis without bond-bending. The Hamiltonian becomes

$$
\begin{aligned}
H_{0}= & \sum \varepsilon_{p} p_{i l, \sigma}^{\dagger} p_{i l, \sigma}+\sum \varepsilon_{d} d_{j m}^{\dagger} d_{j m}-\sum_{n n} V\left[(-1)^{l}\right. \\
& \times \cos \delta \theta_{l} d_{j m}^{\dagger} p_{i l, \uparrow}-\eta_{l}(-1)^{m} e^{i \phi} \\
& \left.\times \sin \delta \theta_{l} d_{j m}^{\dagger} p_{i l, \downarrow}\right]+ \text { H.c. }
\end{aligned}
$$

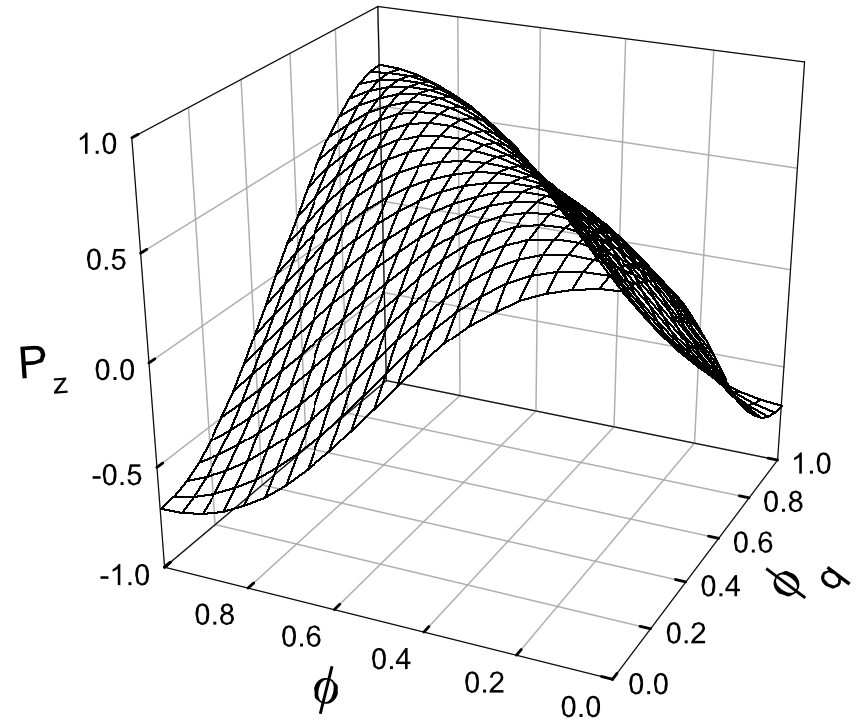

FIG. 3. Polarization $P_{z}$ versus $\phi$ and $\phi_{q}$, where $\phi_{q}$ is the angle between $\vec{q}$ and $x$ axis.

where $d_{j m}=c_{d j m}, \delta \theta_{l}=a_{0} q_{x} / 2, \quad \eta_{l}=1$ for $l=3,4$, and $\delta \theta_{l}$ $=b_{0} q_{y} / 2, \eta_{l}=-1$ for $l=5,6 . a_{0}$ and $b_{0}$ are, respectively, the distance between transition metal ions along the $x$ and $y$ direction. The Hamiltonian in momentum space is

$$
\begin{aligned}
H_{0}= & \sum \varepsilon_{p} p_{l k, \sigma}^{\dagger} p_{l k, \sigma}+\sum \varepsilon_{d} d_{m k}^{\dagger} d_{m k}-\sum_{n n} V\left[(-1)^{l}\right. \\
& \times \cos \delta \theta_{l} e^{i \vec{k} \cdot\left(\vec{R}_{i, l}-\vec{R}_{j, m}\right)} d_{m k}^{\dagger} p_{l k, \uparrow} \\
& \left.-\eta_{l}(-1)^{m} e^{i \phi} \sin \delta \theta_{l} e^{i \vec{k} \cdot\left(\vec{R}_{i, l}-\vec{R}_{j, m}\right)} d_{m k}^{\dagger} p_{l k, \downarrow}\right]+ \text { H.c. }
\end{aligned}
$$

The following is its matrix form:

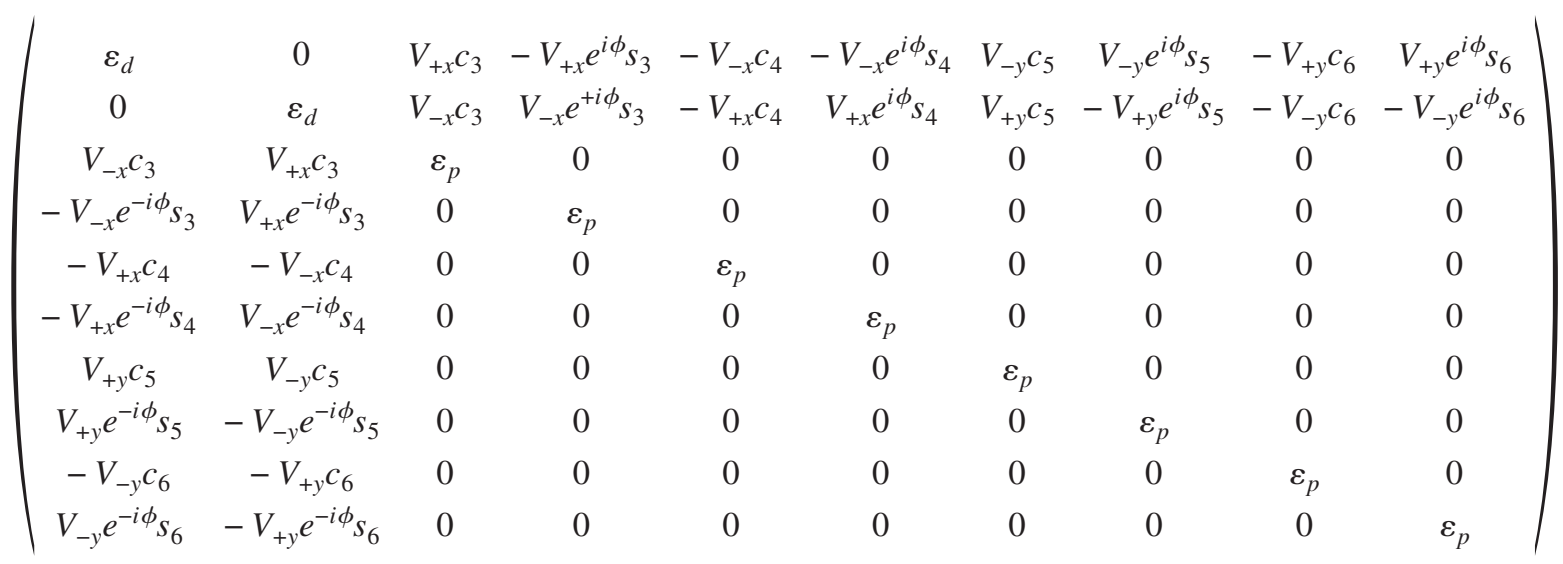

Here, the order of matrix elements is $m=1,2$, and then $l=3 \uparrow, 3 \downarrow, 4 \uparrow, 4 \downarrow$ etc., where $V_{ \pm x}=V e^{ \pm i k_{x} a_{0}}, V_{ \pm y}=V e^{ \pm i k_{y} b_{0}}$ and $s_{i}$ $=\sin \delta \theta_{i}, c_{i}=\cos \delta \theta_{i}$. It can be diagonalized and the eigenvalues are 


$$
\begin{aligned}
& E_{1 \pm}= \frac{\varepsilon_{d}+\varepsilon_{p}}{2} \\
&+\sqrt{\left(\frac{\varepsilon_{d}-\varepsilon_{p}}{2}\right)^{2}+4 V^{2} \pm V^{2}\left|\sum_{n n} \cos \delta \theta_{n n} e^{2 i \vec{k} \cdot R_{n n} \mid}\right|} \\
& E_{2 \pm}= \frac{\varepsilon_{d}+\varepsilon_{p}}{2} \\
&-\sqrt{\left(\frac{\varepsilon_{d}-\varepsilon_{p}}{2}\right)^{2}+4 V^{2} \pm V^{2}\left|\sum_{n n} \cos \delta \theta_{n n} e^{2 i \vec{k} \cdot R_{n n} \mid}\right|}, \\
& E_{3}=\varepsilon_{p}
\end{aligned}
$$

where $\varepsilon_{p}$ is sixfold degenerate and $\vec{R}_{n n}$ and $\delta \theta_{n n}=2 \delta \theta_{l}$ are the position vector and spin angle difference between two nearest neighboring transition element ions. The eigenvectors are

$$
\begin{aligned}
\psi_{1+, \vec{k}, \vec{q}}(\vec{r})= & \sum_{j, m} e^{i \vec{k} \cdot \vec{R}_{j, m} A_{m 1+}}\left[\psi_{d}\left(\vec{r}-\vec{R}_{j, m}\right)\right. \\
& \left.+\sum_{l} \frac{V(-1)^{l} e^{i \vec{k} \cdot \vec{r}_{l m}}}{E_{1+}-\varepsilon_{p}} \psi_{p l}\left(\vec{r}-\vec{R}_{j, l}\right)\right]\left(\begin{array}{c}
\cos \theta_{j, m} \\
e^{i \phi} \sin \theta_{j, m}
\end{array}\right), \\
\psi_{1-, \vec{k}, \vec{q}}(\vec{r})= & \sum_{j, m} e^{i \vec{k} \cdot \vec{R}_{j, m} A_{m 1-}}\left[\psi_{d}\left(\vec{r} \psi_{p l}\right)+\sum_{l} \frac{V(-1)^{l} e^{i \vec{k} \cdot \vec{r}_{l m}}}{E_{1-}-\varepsilon_{p}} \psi_{p l}(\vec{r}\right. \\
& \left.\left.-\vec{R}_{j, l}\right)\right]\left(\begin{array}{c}
\cos \theta_{j, m} \\
e^{i \phi} \sin \theta_{j, m}
\end{array}\right), \\
\psi_{2+, \vec{k}, \vec{q}}(\vec{r})= & \sum_{j, m} e^{i \vec{k} \cdot \vec{R}_{j, m} A_{m 2+}\left[\psi_{d}\left(\vec{r}-\vec{R}_{j, m}\right)\right.} \\
& \left.+\sum_{l} \frac{V(-1)^{l} e^{i \vec{k} \cdot \vec{r}_{l m}}}{E_{2+}-\varepsilon_{p}} \psi_{p l}\left(\vec{r}-\vec{R}_{j, l}\right)\right]\left(\begin{array}{c}
\cos \theta_{j, m} \\
e^{i \phi} \sin \theta_{j, m}
\end{array}\right)
\end{aligned}
$$

$$
\begin{aligned}
\psi_{2-, \vec{k}, \vec{q}}(\vec{r})= & \sum_{j, m} e^{i \vec{k} \cdot \vec{R}_{j, m}} A_{m 2-}\left[\psi_{d}\left(\vec{r}-\vec{R}_{j, m}\right)\right. \\
& \left.+\sum_{l} \frac{V(-1)^{l} e^{i \vec{k} \cdot \vec{r}_{l m}}}{E_{2-}-\varepsilon_{p}} \psi_{p l}\left(\vec{r}-\vec{R}_{j, l}\right)\right]\left(\begin{array}{c}
\cos \theta_{j, m} \\
e^{i \phi} \sin \theta_{j, m}
\end{array}\right)
\end{aligned}
$$

where $A_{m 1(2) \pm}$ are normalization constants, $\vec{r}_{l m}=\vec{r}_{l}-\vec{r}_{m}$, and

$$
\frac{A_{m 1 \pm}}{A_{m 2 \pm}}= \pm \frac{\sum_{n n} \cos \delta \theta_{n n} e^{2 i \vec{k} \cdot \vec{R}_{n n}}}{\left|\sum_{n n} \cos \delta \theta_{n n} e^{2 i \vec{k} \cdot \vec{R}_{n n}}\right|} .
$$

Note that $\psi_{p l}=\psi_{p x}$ for $l=3,4$ and $\psi_{p l}=\psi_{p y}$ for $l=5,6$. The eigenvectors of $\varepsilon_{p}$ do not concern us because they are nonbonding states. If there is no bond-bending, then there are only $\pi$ bonding for $x y$ orbitals. It turns out that the eigenvectors have very similar forms as those in Eqs. (9a)-(9c). They are shown in Appendix.

\section{SPIN-ORBIT INTERACTION AND POLARIZATION}

We now introduce the spin-orbit interaction in Eqs. (4a) and (4b). Its effect can be expressed in the following relations:

$$
\begin{aligned}
& \vec{l} \cdot \vec{s}|x y, \uparrow\rangle=i|z x, \downarrow\rangle+|y z, \downarrow\rangle-i\left|x^{2}-y^{2}, \uparrow\right\rangle, \\
& \vec{l} \cdot \vec{s}|x y, \downarrow\rangle=i|z x, \uparrow\rangle-|y z, \uparrow\rangle+i\left|x^{2}-y^{2}, \downarrow\right\rangle \\
& \vec{l} \cdot \vec{s}\left|x^{2}-y^{2}, \uparrow\right\rangle=|z x, \downarrow\rangle-i|y z, \downarrow\rangle+i|x y, \uparrow\rangle \\
& \vec{l} \cdot \vec{s}\left|x^{2}-y^{2}, \downarrow\right\rangle=-|z x, \uparrow\rangle-i|y z, \uparrow\rangle-i|x y, \downarrow\rangle .
\end{aligned}
$$

Therefore, if the spin-orbit interaction is perturbatively treated, the wave functions $\psi_{d}\left(\vec{r}-\vec{R}_{j, m}\right)\left(\cos \theta_{j, m}|\uparrow\rangle\right.$ $\left.+e^{i \phi} \sin \theta_{j, m}|\downarrow\rangle\right)$ in Eqs. (10a)-(10d) will be replaced by

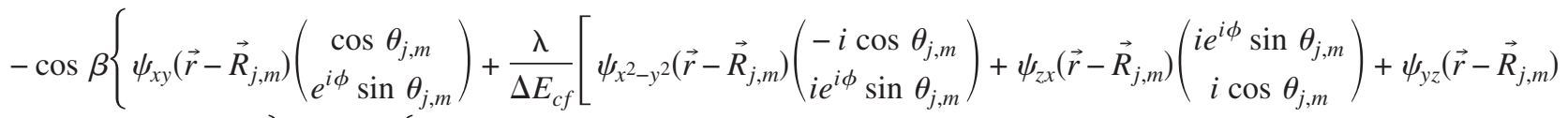

$$
\begin{aligned}
& \left.\left.\times\left(\begin{array}{c}
-e^{i \phi} \sin \theta_{j, m} \\
\cos \theta_{j, m}
\end{array}\right)\right]\right\} \pm \sin \beta\left\{\psi_{x^{2}-y^{2}}\left(\vec{r}-\vec{R}_{j, m}\right)\left(\begin{array}{c}
\cos \theta_{j, m} \\
e^{i \phi} \sin \theta_{j, m}
\end{array}\right)+\frac{\lambda}{\Delta E_{c f}}\left[\begin{array}{c}
\psi_{x y}\left(\vec{r}-\vec{R}_{j, m}\right) \\
\left(i e^{i \phi} \sin \theta_{j, m}\right.
\end{array}\right)+\psi_{z x}\left(\vec{r}-\vec{R}_{j, m}\right)\right. \\
& \left.\left.\times\left(\begin{array}{c}
-e^{i \phi} \sin \theta_{j, m} \\
\cos \theta_{j, m}
\end{array}\right)+\psi_{y z}\left(\vec{r}-\vec{R}_{j, m}\right)\left(\begin{array}{c}
-i e^{i \phi} \sin \theta_{j, m} \\
-i \cos \theta_{j, m}
\end{array}\right)\right]\right\}
\end{aligned}
$$


where $\Delta E_{\mathrm{cf}}$ is the energy difference between $\cos \beta|x y\rangle \mp \sin \beta\left|x^{2}-y^{2}\right\rangle$ in Eq. (2) and $\sin \beta|x y\rangle \pm \cos \beta \mid x^{2}$ $\left.-y^{2}\right\rangle$. Substituting Eq. (13) into Eqs. (10a) $-(10 \mathrm{~d})$, we found that the polarization per unit cell is

$$
\begin{aligned}
\vec{P}_{\vec{q}}= & \langle-e \vec{r}\rangle=\sum_{\mathbf{k}} \int d^{3} \vec{r} \psi_{\vec{k}, \vec{q}}^{*}(\vec{r})(-e \vec{r}) \psi_{\vec{k}, \vec{q}}(\vec{r}) \\
\approx & \frac{8 \sqrt{2} \sin \beta V \lambda}{\pi\left(\varepsilon_{p}-\varepsilon_{d}\right) \Delta E_{c f}}\left(e \rho \hat{e}_{z}\right)\left[\cos \phi \sin \left(a_{0} q_{x} / 2\right)\right. \\
& \left.+\sin \phi \sin \left(b_{0} q_{y} / 2\right)\right],
\end{aligned}
$$

where

$$
\begin{aligned}
\rho & \approx-\int d^{3} \vec{r} \psi_{z x}^{*}(\vec{r}) z \psi_{p x}\left(\vec{r}-a_{0} \hat{e}_{x} / 2\right) \\
& \approx-\int d^{3} \vec{r} \psi_{y z}^{*}(\vec{r}) z \psi_{p y}\left(\vec{r}-b_{0} \hat{e}_{y} / 2\right),
\end{aligned}
$$

with $a_{0}$ and $b_{0}$ being the lattice constants without bondbending. Another way of writing them should be $\vec{a}_{0}=\left(\vec{R}_{j+1}\right.$ $\left.-\vec{R}_{j}\right) / 2$ and $\vec{b}_{0}=\left(\vec{R}_{j+1}-\vec{R}_{j}\right) / 2$ for site $j+1$ situated at $x$ or $y$ direction away from site $j$. There are ten bands in our calculation. The main contribution comes from the topmost occupied band. We have also made the approximation $E_{n+} \approx \varepsilon_{p}$, which takes the advantage of the fact that $V \ll\left|\varepsilon_{p}-\varepsilon_{d}\right|$. If even number of bands are occupied, the polarization will be much smaller. The polarization produced by two bands tends to cancel each other. As a result, there is an extra factor of $4 V^{2} /\left(\varepsilon_{p}-\varepsilon_{d}\right)^{2}$, which comes from the denominator of Eqs. (10a)-(10d),

$$
\begin{aligned}
\vec{P}_{\vec{q}} \approx & \frac{32 \sqrt{2} \sin \beta V^{3} \lambda}{\pi\left(\varepsilon_{p}-\varepsilon_{d}\right)^{3} \Delta E_{c f}}\left(e \rho \hat{e}_{z}\right)\left[\cos \phi \sin \left(a_{0} q_{x} / 2\right)\right. \\
& \left.+\sin \phi \sin \left(b_{0} q_{y} / 2\right)\right] .
\end{aligned}
$$

To more clearly see how polarization and $\vec{q}$ are related, let $U_{P}$ be the space inversion operator. We then have

$$
U_{P} \psi_{\vec{k}, \vec{q}}(\vec{r})=\psi_{-\vec{k},-\vec{q}}(\vec{r}) .
$$

Under the space inversion, the displacements of oxygen atoms and hence, $\alpha, \beta$, and $V$ change sign under inversion,

$$
\vec{P}_{\vec{q}}=-e\left\langle U_{P} U_{P} \vec{r} U_{P} U_{P}\right\rangle=-\vec{P}_{-\vec{q}}
$$

and the polarization is an odd function of $\vec{q}$. The form of sine function in Eqs. (14) and (16) seems to be a natural form. In the continuum limit $\left(a_{0} \approx b_{0} \rightarrow 0\right)$ or the long wavelength limit $(\vec{q} \rightarrow 0)$, we found

$$
\vec{P}_{\vec{q}} \sim 0.01 e \rho a_{0} \hat{e}_{z}\left(q_{x} \cos \phi+q_{y} \sin \phi\right) \sim 0.01 e \rho a_{0} \vec{q} \times \hat{h}
$$

where $\hat{h}$ is the helix axis unit vector. We have assumed that $q_{z}=0$ since our calculation was performed on $x y$ plane. If one recognizes that the true meaning of $\hat{e}_{12}$ is the spatial direction alone which the spins propagate, i.e., $\vec{q}$, he can see how the form $\vec{P} \sim e l \hat{e}_{12} \times\left(\hat{e}_{1} \times \hat{e}_{2}\right)$ given by KNB can be transformed in the presence of magnetic orders.

\section{DISCUSSION}

In order to see how magnetic orders and electric order are coupled, let us go back to the original work of Moriya. ${ }^{19} \mathrm{He}$ derived the following expression:

$$
H_{\mathrm{DM}}=\sum_{N} \vec{D}_{N, N-1} \cdot\left(\vec{S}_{N} \times \vec{S}_{N-1}\right)
$$

where

$$
\begin{aligned}
\vec{D}_{N, N-1}= & i \lambda \sum \frac{J\left(n, n^{\prime}, m, n^{\prime}\right)\left\langle n\left|\vec{l}_{i}\right| m\right\rangle}{E_{n}-E_{m}} \\
& -i \lambda \sum \frac{J\left(n, n^{\prime}, n, m^{\prime}\right)\left\langle n^{\prime}\left|\vec{l}_{j}\right| m^{\prime}\right\rangle}{E_{n^{\prime}}-E_{m^{\prime}}} .
\end{aligned}
$$

Here, $J\left(n, n^{\prime}, m, m^{\prime}\right)$ is the exchange interaction strength and $\vec{l}_{i}$ denotes the angular momentum of the electron at site $i$.

In our starting Hamiltonian, the exchange interaction comes from the charge transfer energy $\varepsilon_{p}-\varepsilon_{d}$ and hybridization energy $V .{ }^{27}$ Combined with the spin-orbit interaction in Eq. (4b), DM interaction is clearly present in the system we considered. We can recast the wave functions we previously got in a form similar to Moriya's by treating the hybridization energy and spin-orbit interaction as perturbations,

$$
\vec{P}_{\vec{q}}=-e \sum_{n, m, l} \frac{\left\langle m\left|H_{V}\right| n\right\rangle \lambda\langle n|\vec{l}| l\rangle \cdot\left\langle\sigma_{n}|\vec{s}| \sigma_{l}\right\rangle}{\left(\varepsilon_{d}-\varepsilon_{p}\right) \Delta E_{\mathrm{cf}}}\left\langle l, \sigma_{l}|\vec{r}| m, \sigma_{m}\right\rangle+\text { c.c. }
$$

where $H_{V}$ is the hybridization energy [the third and fourth terms of Eq. (4a)]. $|l\rangle$ and $\left|\sigma_{l}\right\rangle$ are, respectively, the spatial and spin part of an intermediate state. $|n\rangle$ and $|l\rangle$ are states of $d$ orbitals and $|m\rangle p$ orbitals. $\left\langle n\left|H_{V}\right| m\right\rangle /\left(\varepsilon_{d}-\varepsilon_{p}\right)$ is the exchange part where $H_{V}$ also contains information of spins. $\lambda\langle l|\vec{l}| n\rangle \cdot\left\langle\sigma_{l}|\vec{s}| \sigma_{n}\right\rangle$ is the spin-orbit coupling. Equation (22) is also applicable to the situations without bond-bending. In that case, the $\pi$ bonding will be considered as they were in $\mathrm{KNB}$ original work and $H_{V}$ is the third term of Eq. (A1). One can see that Eq. (22) has the same origin as $H_{\mathrm{DM}}$ in Eqs. (20) and (21). However, we have to note that the helical spin configuration is not caused by DM interaction whose strength is too small. Rather, it can be due to the nextnearest-neighbor hybridization, as shown in Ref. 23.

It is easier to analyze with the following form:

$$
\begin{aligned}
P_{\vec{q}, k} & =-e \sum_{n, m, l, j} \\
& \times \frac{\left\langle m\left|H_{V}\right| n\right\rangle \lambda\left\langle n\left|\varepsilon_{h i j} r_{h} p_{i}\right| l\right\rangle\left\langle\sigma_{n}\left|s_{j}\right| \sigma_{l}\right\rangle\left\langle l, \sigma_{l}\left|r_{k}\right| m, \sigma_{m}\right\rangle}{\left(\varepsilon_{d}-\varepsilon_{p}\right) \Delta E_{\mathrm{cf}}} \\
& + \text { c.c. }
\end{aligned}
$$

where $\varepsilon_{h i j}$ is the antisymmetric Levi-Cevita symbol and $r_{k}$ is the $k$ th component of $\vec{r}$ in space. We consider the mirror symmetry of above equation. $H_{V}$ may change sign under mirror reflection operation because of the orbital wave functions involved. It is also related to the direction of the displacements of oxygen atoms. For example, the $(p d \pi)$ part of $E_{y, x y}$ and $(p d \sigma)$ of $E_{x, x^{2}-y^{2}}$ (in the Slater-Koster notation) with 
bond along the $x$ direction change sign if one makes the operation $x \rightarrow-x$. On the other hand, the $(p d \sigma)$ part of $E_{x, x y}$ (due to bond-bending) does not change sign if one makes the operation $x \rightarrow-x$ or $y \rightarrow-y$. In Sec. IV, we calculated the polarization of a planar crystal. Now, we consider a more general case of orthorhombic structure and helical spin configuration. It is easier to catch the essence if one considers the $(p d \pi)$ part of $E_{y, x y}$ or the $(p d \sigma)$ part of $E_{x, x^{2}-y^{2}}$ of $H_{V}$, which is applicable to KNB's original work and to the $x^{2}$ $-y^{2}$ orbital part of our work, respectively. Assuming the bond direction of $H_{V}$ is in the $m$ direction, then polarization is finite for either $k=h, m=i$ or $k=i, m=h$. As a result, Eq. (23) can be simplified as

$$
\begin{aligned}
P_{\vec{q}, k}= & -2 e \sum_{n, m, l, j} \frac{\left\langle m\left|H_{V, i}\right| n\right\rangle \lambda\left\langle n\left|l_{j}\right| l\right\rangle\left\langle\sigma_{n}\left|s_{j}\right| \sigma_{l}\right\rangle}{\left(\varepsilon_{d}-\varepsilon_{p}\right)} \frac{\left\langle l, \sigma_{l}\left|r_{k}\right| m, \sigma_{m}\right\rangle}{\Delta E_{\mathrm{cf}}} \\
& + \text { c.c. }
\end{aligned}
$$

where $H_{V, i}$ denotes the hybridization bond along the $i$ direction and $i-k$ are cyclic.

The spin part needs more attention. $\left\langle\sigma_{n}\left|s_{j}\right| \sigma_{l}\right\rangle$ give rise to a spin state $\left|\sigma_{l}\right\rangle$ different from the original state $\left|\sigma_{n}\right\rangle$ and $\left\langle\sigma_{l} \mid \sigma_{m}\right\rangle$ gives rise to a interesting contribution. Only the imaginary part needs to be considered because $\left\langle l\left|l_{j}\right| n\right\rangle$ is imaginary,

$$
\begin{aligned}
\operatorname{Im}\left(\left\langle\sigma_{n}\left|s_{x}\right| \sigma_{l}\right\rangle\left\langle\sigma_{l, x} \mid \sigma_{n}\right\rangle\right) & =\sin \phi \sin \left(\vec{q} \cdot \vec{R}_{N, N-1} / 2\right) \\
& =\left.\left(\vec{s}_{N} \times \vec{s}_{N-1}\right)\right|_{x}, \\
\operatorname{Im}\left(\left\langle\sigma_{n}\left|s_{y}\right| \sigma_{l}\right\rangle\left\langle\sigma_{l, y} \mid \sigma_{n}\right\rangle\right) & =-\cos \phi \sin \left(\vec{q} \cdot \vec{R}_{N, N-1} / 2\right) \\
& =\left.\left(\vec{s}_{N} \times \vec{s}_{N-1}\right)\right|_{y},
\end{aligned}
$$

for the spin configuration in Eq. (1). Here, the additional subscript $j$ of $\left|\sigma_{l, j}\right\rangle$ denotes that it comes from $\left\langle\sigma_{n}\left|s_{j}\right| \sigma_{l}\right\rangle$. The right hand sides of Eqs. (24) are very similar to respective components of $\vec{S}_{N} \times \vec{S}_{N-1}$ which appears in $H_{\mathrm{DM}}$. The difference is in the arguments of sin functions. The factor $1 / 2$ arises because in our model, it is the hybridization electrons that mediate the exchange interaction, while in the original DM interaction, it is direct exchange. Substituting Eqs. (25a) and (25b) into Eq. (24), we found that

$P_{\vec{q}, k}=-2 e \sum_{n, m, l, j} \frac{\lambda\left\langle m\left|H_{V, i}\right| n\right\rangle}{\left(\varepsilon_{d}-\varepsilon_{p}\right)}\left\langle n\left|l_{j}\right| l\right\rangle\left(\vec{s}_{N} \times \vec{s}_{N-1}\right) \mid \frac{\left\langle l\left|r_{k}\right| m\right\rangle}{\Delta E_{c f}}+$ c.c.

The connection between the electric polarization and spin current can now be established. A common definition of the spin current is

$$
\widetilde{j_{i}^{j}}=\left.\frac{t}{4 i \hbar} \sum_{N}\left(d_{\mathbf{R}_{N}+\mathbf{a}}^{+} \sigma_{j} d_{\mathbf{R}_{N}}-d_{\mathbf{R}_{N}}^{+} \sigma_{j} d_{\mathbf{R}_{N}+\mathbf{a}}\right) \vec{a}\right|_{i},
$$

where $\vec{a}$ is the lattice vector of length $a_{0}$ in the direction of $i$ and in our case, $t \approx V^{2} /\left|\varepsilon_{d p}\right|$. Note that the electrons hop along the direction of $\vec{a}$. Equation (27) can be derived by discretizing the form $i \hbar\left[\psi^{+} \sigma_{j} \partial_{i} \psi-\left(\partial_{i} \psi^{+}\right) \sigma_{j} \psi\right] / 4 m$. The form in Eq. (27) manifests itself in helical spin configuration. We calculated its expectation value with the state in Eq. (2) and found that

$$
\begin{gathered}
\left\langle\widetilde{j}_{x}^{y}\right\rangle=\frac{t a_{0}}{2 \hbar} \cos \phi \sin \left(q_{x} a_{0} / 2\right)=-\left.\frac{t a_{0}}{2 \hbar}\left(\vec{s}_{N} \times \vec{s}_{N-1}\right)\right|_{y}, \\
\left\langle\widetilde{j}_{y}^{x}\right\rangle=-\frac{t a_{0}}{2 \hbar} \sin \phi \sin \left(q_{y} a_{0} / 2\right)=-\left.\frac{t a_{0}}{2 \hbar}\left(\vec{s}_{N} \times \vec{s}_{N-1}\right)\right|_{x} .
\end{gathered}
$$

The results are the same as those in Eqs. (25a) and (25b). Introducing the $\mathrm{SU}(2)$ vector potential ${ }^{28} \mathcal{A}_{j}^{i}$ $=-\varepsilon_{i j k} \hbar E_{k} / 4 m_{e} c^{2}$, where $m_{e}$ is the electron mass, the DM interaction can be rewritten as

$$
H_{\mathrm{DM}}=e \sum \mathcal{A}_{j}^{i} \widetilde{\mathrm{J}}_{i}^{j},
$$

where

$$
\mathcal{A}_{j}^{i}=-\frac{2 J \lambda}{e t a_{0} \Delta E_{c f}} e_{B, i} l_{j},
$$

is the guage field coupled to the spin current. It comes from the term $\vec{E} \cdot(\vec{p} \times \vec{\sigma})$ in Pauli's equation (see for example, Ref. 28). The polarization results from the perturbation of $H_{D M}$,

$$
P_{\vec{q}, k}=-e \sum_{M} \frac{\left\langle M\left|H_{\mathrm{DM}}\right| 0\right\rangle}{E_{0 M}}\left\langle 0\left|r_{k}\right| M\right\rangle,
$$

where $|0\rangle$, the ground state, has a component $\left(V / \varepsilon_{d p}\right)\left|\psi_{p}\right\rangle$. If we take $E_{0 M}$ to be the exchange energy $J$, then Eq. (25a) and (25b) and Eq. (30) are equivalent in view of Eqs. (26), (28a), (28b), and (30).

Now, we can more clearly see what the origin of internal electric field $E_{k}$ is. From Eq. (30), we found

$$
\vec{E}=\frac{8 m_{e} c^{2} J \lambda}{e t a_{0} \Delta E_{\mathrm{cf}}} \hat{e}_{B} \times \vec{l},
$$

where the factor $m_{e} c^{2}$ will be canceled by its inverse in $\lambda$. The small factor $J / t$ arises because of the cancellation between different bands [see also Eqs. (14) and (16)]. The electric field originates from the perturbation of spin-orbit interaction. The spin-orbit interaction changes the angular dependence of the $d$-orbital wave functions and hence, the electron density, via the hybridization of $d$ and $p$ orbitals. This effect can be interpreted as the result of an internal electric field. However, the electric field thus created does not necessarily give rise to net electric polarization, as shown in Fig. 4. Certain environments are more advantageous than others. As one can see from Eqs. (20), (25a), (25b), (28a), and (28b), the helical spin configuration is apt to provide spin current, net electric polarization, and multiferroics.

The magnitude of polarization is also important. If there are odd number of filled bands, then the polarization is of the order $\vec{P}_{\vec{q}} / \Omega$, where $\Omega \sim 250 \AA^{3}$ is the volume of a unit cell and Eq. (14) is used. If we take $\left|\varepsilon_{p}-\varepsilon_{d}\right| \sim 2 \mathrm{eV}, \Delta E_{\mathrm{cf}}$ $\sim 2.0 \mathrm{eV}, \quad V=(\sqrt{3} / 2) \sin (\alpha-\beta) \sin (\alpha / 2) V(p d \sigma) \sim 0.2 \mathrm{eV}, \lambda$ $\sim 0.05 \mathrm{eV}$, and $\sin \beta \sim 0.1$, then $P \sim 10 \mu C / \mathrm{m}^{2}$ for $\rho$ 

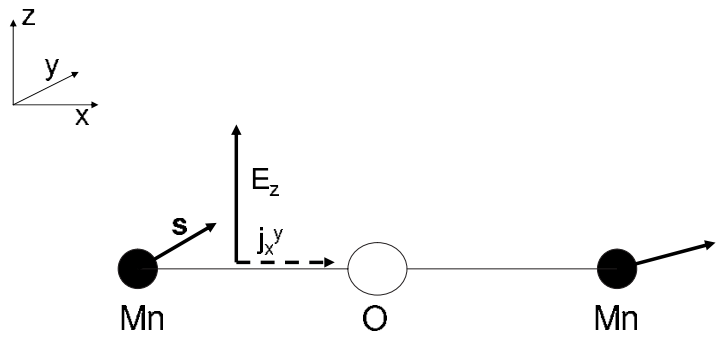

FIG. 4. A spin current along the Mn-O-Mn bond ( $y$ component of spins moving in the $x$ direction.) It can be coupled to the $z$ component of electric field and induce polarization $P_{z}$.

$\sim 0.1 \AA$. The bond-bending activated hybridization $V$ is, in general, greater than $V(p d \pi)$ and $\rho \sin \beta / I$, where $I$ is defined in Eq. (A6), is of the order $V(p d \sigma) \sin \beta / V(p d \pi) \sim 1$ for $\beta \approx \pi / 12$. Hence, bond-bending gives larger polarization in many oxides. For example, $\alpha \approx 5 \pi / 6$ in manganites ${ }^{26}$ and in compounds such as $\mathrm{Ni}_{3} \mathrm{~V}_{2} \mathrm{O}_{8}$, the bond angle of Ni-O-Ni $\alpha$ can be as small as $\pi / 2{ }^{6}$ Hence, $\alpha$ and $\beta$ may both be large and the environment is favorable to ferroelectricity. Furthermore, $\rho \sim 0.1 \AA$ can be very well an underestimation because the $O^{2-}$ has a much larger radius than a neutral oxygen atom. If all things considered, $P$ can be an order of magnitude greater than previously estimated. Atomic displacement can also enhance electric polarization by destroying cancellation. However, if it has its own wave vector and it is not commensurate with $\vec{q}$, then there is no net polarization.

In conclusion, we have analyzed the conditions for the emergence of ferroelectricity due to magnetic orders. We found a simple relation between its wave vector $\vec{q}$ and polarization. Furthermore, the physical picture of the coupling between magnetic orders and ferroelectricity is made clear. Multiferroics is created by a generalized version of Dzyaloshinskii-Moriya interaction in the environment of certain spin orders, preferably helical. Above findings can also be applied to systems without bond-bending but with $\pi$-bond hybridization. The bond-bending tends to enhance polarization and it may be important for certain compounds.

\section{ACKNOWLEDGMENTS}

The author benefited from the activities of "quantum novel phenomena in condensed matter" focus groups of NCTS, Taiwan and discussion with S. Maekawa. This work is supported in part by the National Science Council of Taiwan under Contract No. NSC 95-2112-M-002-048-MY3.

\section{APPENDIX}

In this appendix, we present the eigenvalues and eigenvectors of the $\pi$-bonding systems. It is simpler because there are only one transition element ion (thus the dropping of the index $m$ ) and two oxygen atoms in the basis. We consider a planar crystal on the $x y$ plane. For bonds along $x(y)$ direction, $p_{y(x)}$ orbital of the oxygen atoms, and the $x y$ orbital of the transition element ions form $\pi$ bond. The $p_{z}$ orbitals are ignored because they do not give rise to polarization in the $z$ direction where orbitals are considered. Thus, we have the Hamiltonian

$$
\begin{aligned}
H_{0}= & \sum \varepsilon_{p} c_{p i l, \sigma}^{\dagger} c_{p i l, \sigma}+\sum \varepsilon_{d} c_{d j}^{\dagger} c_{d j}-\sum_{n . n .} V^{\prime}(-1)^{l} \\
& \times\left[\cos \theta_{j} c_{d j}^{\dagger} c_{p i l, \uparrow}+e^{i \phi} \sin \theta_{j} c_{d j}^{\dagger} c_{p i l, \downarrow}\right]+\text { H.c. },
\end{aligned}
$$

where $V^{\prime}=V(p d \pi)$ and $l=1,2$ for the oxygen atoms on the $x$ axis and $y$ axis respectively. Making a transformation similar to that in Eqs. (5a) and (5b), we obtain the Hamiltonian in momentum space

$$
\begin{aligned}
H_{0}= & \sum \varepsilon_{p} p_{l k, \sigma}^{\dagger} p_{l k, \sigma}+\sum \varepsilon_{d} d_{k}^{\dagger} d_{k}-\sum V_{n . n .} V^{\prime}(-1)^{l} \\
& \times\left[\cos \delta \theta_{l} e^{\left(\vec{R}_{i, l}-\vec{R}_{j}\right)} d_{k}^{\dagger} p_{l k, \uparrow}-e^{i \phi} \sin \delta \theta_{l} e^{i \vec{k} \cdot\left(\vec{R}_{i, l}-\vec{R}_{j}\right)} d_{k}^{\dagger} p_{l k, \uparrow}\right] \\
& + \text { H.c. }
\end{aligned}
$$

Now, $\delta \theta_{l=3,4}=q_{x} a_{0} / 2$ and $\delta \theta_{l=5,6}=q_{y} b_{0}$. We can solve for eigenvalues,

$$
\begin{aligned}
E_{1 \pm}= & \frac{\varepsilon_{d}+\varepsilon_{p}}{2} \\
& +\sqrt{\left(\frac{\varepsilon_{d}-\varepsilon_{p}}{2}\right)^{2}+4 V^{\prime 2} \pm V^{\prime 2}\left|\sum_{l} \cos 2 \delta \theta_{l} e^{2 i \vec{k} \cdot \vec{r}_{l 1}}\right|},
\end{aligned}
$$

$$
\begin{aligned}
E_{2 \pm}= & \frac{\varepsilon_{d}+\varepsilon_{p}}{2} \\
& -\sqrt{\left(\frac{\varepsilon_{d}-\varepsilon_{p}}{2}\right)^{2}+4 V^{\prime 2} \pm V^{\prime 2}\left|\sum_{l} \cos 2 \delta \theta_{l} e^{2 i \vec{k} \cdot \vec{r}_{l 1}}\right|},
\end{aligned}
$$

$$
E=\varepsilon_{p},
$$

where $\varepsilon_{p}$ is twofold degenerate. The eigenvectors are

$$
\begin{aligned}
& \psi_{1+, \vec{k}, \vec{q}}^{\prime}(\vec{r})=\sum_{j} e^{i \vec{k} \cdot \vec{R}_{j}} A_{1+}\left[\psi_{d}\left(\vec{r}-\vec{R}_{j}\right)+\sum_{l} \frac{V^{\prime}(-1)^{l} e^{i \vec{k} \cdot \vec{r}_{l}}}{E_{1+}-\varepsilon_{p}} \psi_{p l}(\vec{r}\right. \\
& \left.\left.-\vec{R}_{j, l}\right)\right]\left(\begin{array}{c}
\cos \theta_{j} \\
e^{i \phi} \sin \theta_{j}
\end{array}\right), \\
& \psi_{1-, \vec{k}, \vec{q}}^{\prime}(\vec{r})=\sum_{j} e^{i \vec{k} \cdot \vec{R}_{j}} A_{1-}\left[\psi_{d}\left(\vec{r}-\vec{R}_{j}\right)+\sum_{l} \frac{V^{\prime}(-1)^{l} e^{i \vec{k} \cdot \vec{r}_{l}}}{E_{1-}-\varepsilon_{p}} \psi_{p l}(\vec{r}\right. \\
& \left.\left.-\vec{R}_{j, l}\right)\right]\left(\begin{array}{c}
\cos \theta_{j} \\
e^{i \phi} \sin \theta_{j}
\end{array}\right) \\
& \psi_{2+, \vec{k}, \vec{q}}^{\prime}(\vec{r})=\sum_{j} e^{i \vec{k} \cdot \vec{R}_{j}} A_{2+}\left[\psi_{d}\left(\vec{r}-\vec{R}_{j}\right)+\sum_{l} \frac{V^{\prime}(-1)^{l} e^{i \vec{k} \cdot \vec{r}_{l}}}{E_{2+}-\varepsilon_{p}} \psi_{p l}(\vec{r}\right. \\
& \left.\left.-\vec{R}_{j, l}\right)\right]\left(\begin{array}{c}
\cos \theta_{j} \\
e^{i \phi} \sin \theta_{j}
\end{array}\right),
\end{aligned}
$$




$$
\begin{aligned}
\psi_{2-, \vec{k}, \vec{q}}^{\prime}(\vec{r})= & \sum_{j} e^{i \vec{k} \cdot \vec{R}_{j}} A_{2-}\left[\psi_{d}\left(\vec{r}-\vec{R}_{j}\right)+\sum_{l} \frac{V^{\prime}(-1)^{l} e^{i \vec{k} \cdot \vec{r}_{l}}}{E_{2-}-\varepsilon_{p}} \psi_{p l}(\vec{r}\right. \\
& \left.\left.-\vec{R}_{j, l}\right)\right]\left(\begin{array}{c}
\cos \theta_{j} \\
e^{i \phi} \sin \theta_{j}
\end{array}\right),
\end{aligned}
$$

where $A_{1 \pm}$ and $A_{2 \pm}$ are normalization constants. One can easily see the similarity between the wave functions with or without bond-bending. The polarization can be computed as Eq. (13). The result is

$$
\begin{aligned}
\vec{P}_{\vec{q}}= & \langle-e \vec{r}\rangle \approx \frac{4 \lambda V^{\prime}}{\pi\left(\varepsilon_{p}-\varepsilon_{d}\right) \Delta E_{c f}^{\prime}}\left(e I \hat{e}_{z}\right)\left[\cos \phi \sin \left(a_{0} q_{x} / 2\right)\right. \\
& \left.+\sin \phi \sin \left(b_{0} q_{y} / 2\right)\right]
\end{aligned}
$$

where $\Delta E_{\mathrm{cf}}^{\prime}$ is the energy difference between $t_{2 g}$ states and $e_{g}$ states and

$I=\int d^{3} \vec{r} \psi_{y z}^{*}(\vec{r}) z \psi_{p y}\left(\vec{r}-a_{0} \hat{e}_{x} / 2\right)=\int d^{3} \vec{r} \psi_{z x}^{*}(\vec{r}) z \psi_{p x}\left(\vec{r}-b_{0} \hat{e}_{y} / 2\right)$
${ }^{1}$ Z. J. Huang, Y. Cao, Y. Y. Sun, Y. Y. Xue, and C. W. Chu, Phys. Rev. B 56, 2623 (1997).

${ }^{2}$ B. Lorenz, Y. Q. Wang, Y. Y. Sun, and C. W. Chu, Phys. Rev. B 70, 212412 (1997).

${ }^{3}$ T. Kimura, T. Goto, H. Shintani, K. Ishizaka, T. Arima, and Y. Tokura, Nature (London) 426, 55 (2003).

${ }^{4}$ T. Kimura, G. Lawes, T. Goto, Y. Tokura, and A. P. Ramirez, Phys. Rev. B 71, 224425 (2005).

${ }^{5}$ L. C. Chapon, G. R. Blake, M. J. Gutmann, S. Park, N. Hur, P. G. Radaelli, and S-W. Cheong, Phys. Rev. Lett. 93, 177402 (2004).

${ }^{6}$ M. Kenzelmann, A. B. Harris, A. Aharony, O. Entin-Wohlman, T. Yildirim, Q. Huang, S. Park, G. Lawes, C. Broholm, N. Rogado, R. J. Cava, K. H. Kim, G. Jorge, and A. P. Ramirez, Phys. Rev. B 74, 014429 (2006).

${ }^{7}$ Y. Yamasaki, H. Sagayama, and T. Goto, M. Matsuura, K. Hirota, T. Arima, and Y. Tokura, Phys. Rev. Lett. 98, 147204 (2007).

${ }^{8}$ M. Kenzelmann, G. Lawes, A. B. Harris, G. Gasparovic, C. Broholm, A. P. Ramirez, G. A. Jorge, M. Jaime, S. Park, Q. Huang, A. Ya. Shapiro, and L. A. Demianets, Phys. Rev. Lett. 98, 267205 (2007).

${ }^{9}$ M. Kenzelmann, A. B. Harris, S. Jonas, C. Broholm, J. Schefer, S. B. Kim, C. L. Zhang, S.-W. Cheong, O. P. Vajk, and J. W. Lynn, Phys. Rev. Lett. 95, 087206 (2005).

${ }^{10}$ Maxim Mostovoy, Phys. Rev. Lett. 96, 067601 (2006).

${ }^{11}$ I. E. Chupis, arXiv:cond-mat/0702636 (unpublished).

${ }^{12}$ Ivan A. Sergienko and E. Dagotto, Phys. Rev. B 73, 094434 (2006).
${ }^{13}$ Ivan A. Sergienko, Cengiz Sen, and Elbio Dagotto, Phys. Rev. Lett. 97, 227204 (2006).

${ }^{14}$ E. R. Sánchez Guajardo, arXiv:cond-mat/0608300 (unpublished).

${ }^{15}$ Seongsu Lee, A. Pirogov, Jung Hoon, Han, J.-G. Park, A. Hoshikawa, and T. Kamiyama, Phys. Rev. B 71, 180413 (2005).

${ }^{16}$ Hosho Katsura, Naoto Nagaosa, and Aleander V. Balatsky, Phys. Rev. Lett. 95, 057205 (2005).

${ }^{17}$ Although the use of spin current may raise the issue of whether it is well defined in the presence of spin-orbit interaction, we nevertheless still use this term as no ambiguity involved here

${ }^{18}$ I. Dzyaloshinskii, J. Phys. Chem. Solids 4, 241 (1958).

${ }^{19}$ T. Moriya, Phys. Rev. 120, 91 (1960).

${ }^{20}$ Y. Aharonov and A. Casher, Phys. Rev. Lett. 53, 319 (1984).

${ }^{21}$ Chenglong Jia, Shigeki Onoda, Naoto Nagaosa, and Jung Hoon Han, Phys. Rev. B 74, 224444 (2006).

${ }^{22}$ C. D. Hu, Phys. Rev. B 75, 172106 (2007).

${ }^{23}$ Maxim Mostovoy, Phys. Rev. Lett. 94, 137205 (2005).

${ }^{24}$ T. Kimura, S. Ishihara, H. Shintani, T. Arima, K. T. Takahashi, K. Ishizaka, and Y. Tokura, Phys. Rev. B 68, 060403(R) (2003).

${ }^{25}$ J. C. Slater and G. F. Koster, Phys. Rev. 94, 1498 (1954).

${ }^{26}$ T. Kimura, S. Ishihara, H. Shintani, T. Arima, K. T. Takahashi, K. Ishizaka, and Y. Tokura, Phys. Rev. B 68, 060403(R) (2003).

${ }^{27}$ According to the calculation of Ref. 21, introducing the on-site Coulomb repulsion will not change qualitatively the physical, picture.

${ }^{28}$ B. W. A. Leurs, Z. Nazario, D. I. Santiago, and J. Zaanen, arXiv:0705.2953 (unpublished). 